\title{
Pure Titanium Foil Hybrid Surface Modification Using Ultraviolet Laser Micromachining
}

\author{
Chih-Chung Yang*, Han-Chao Chang*, Yu-Chen Hsien*, Shih-Feng Tseng ${ }^{* *}$, Keh-moh Lin ${ }^{* * *}$, Donyau Chiang*, Kuo-Cheng \\ Huang*, Wen-Tse Hsiao* \\ * Instrument Technology Research Center, National Applied Research Laboratories, Taiwan, 20, \\ R\&D Rd. VI, Hsinchu Science Park, Hsinchu, 30076, Taiwan \\ ** Department of Mechanical Engineering, National Taipei University of Technology, Taiwan, 1, Sec. \\ 3, Zhongxiao E. Rd., Taipei, 10608, Taiwan \\ *** Department of Mechanical Engineering, Southern Taiwan University of Science and Technology, Taiwan, 1, Nan- \\ Tai St., Yungkang Dist., Tainan, 71005, Taiwan \\ E-mail: wentse@narlabs.org.tw
}

\begin{abstract}
This study proposes a hybrid laser method for modifying the surface of a pure titanium foil (thickness: $100 \mu \mathrm{m}$ ) using nanosecond ultraviolet (UV) laser pulses. Four surface treatment methods, namely nontreatment, microhole drilling, surface milling, and microhole drilling combined with surface milling, were used, and the effects of contact angle, surface energy, roughness, and residual height were studied. The characteristics of the surface-modified foil specimens were systemically analyzed using a three-dimensional confocal laser scanning microscope to measure the surface micrograph and roughness of patterns. The contact angle variations between different droplets on the textured surface were measured using an FTA 188 video contact angle analyzer. In this study, the optimal laser fluence and scanning speed for the laser surface milling process were $56.6 \mathrm{~J} / \mathrm{cm}^{2}$ and $1500 \mathrm{~mm} / \mathrm{s}$, respectively, which could provide a contact angle of $12.57^{\circ}$, signifying a foil surface with satisfactory hydrophilic quality. This study successfully used a UV laser combined with a highspeed galvanometric scanning unit to generate different microstructures on the surfaces of pure titanium foil specimens. In the future, the proposed method can be applied for research related to the adhesive quality of biological cells.
\end{abstract}

DOI: 10.2961/jlmn.2018.03.0012

Keywords: micro-hole drilling (Chief Editor's comment, Q1), laser milling, contact angle, titanium foil, hydrophilic

\section{Introduction (Editor's and Reviewer \#2, Q1)}

Titanium alloys exhibit high strength, corrosion resistance, low elastic modulus, satisfactory heat resistance, satisfactory low-temperature performance, high biocompatibility, low coefficient of heat transfer, and nonmagnetic properties [1,2]; moreover, they can be implanted in body tissue in vivo and can be combined with each other at various levels of biological organization to enhance their effectiveness. Therefore, titanium alloys are widely used in various applications including bone plates, implants, crowns, and artificial joints. Among titanium alloys, titanium meshes have been successfully applied to spinal fusion surgery and oral and craniofacial reconstruction. Because these materials have shown superior acceptability by human tissues compared with other metals under diverse circumstances, they are considered ideal materials [1].

To obtain (Editor's and Reviewer \#2, Q2) satisfactory tissue growth and improved bonding performance, some studies have proposed surface modification methods. Surface modification can improve biocompatibility, in addition to enhancing antibacterial properties, increasing surface lubricity, improving wetting, wear, and corrosion resistance, increasing service life, and reducing friction between tissues and organisms. The relevant literature reports several surface treatment methods including lithography, dry/wet etching, coating, and diamond dicing processes [3-6]. To reduce production costs and increase processing speed, laser micro-/nano-machining processes involving techniques such as laser ablation, laser milling, and laser deposition have been effectively used to manufacture components with enhanced machined surface properties $[7,8]$. Laser material processing is an advanced manufacturing process with characteristics such as noncontact processing, lack of tool wear, little break of chipping, high accuracy, high resolution, high speed, and low thermal stress. Scholars have used different polarization technologies to fabricate periodic structures on the surfaces of materials. For example, Zheng et al. [9] used a femtosecond laser source combined with an interferometry method to fabricate periodic structures on fused silica and crystalline silicon materials. Moreover, a p-polarization laser beam was used to produce microchannels having a width and spacing of 20 $\mu \mathrm{m}$ with no microcracks. Hee et al. [10] used 150-fs Tisapphire laser pulses with four polarization combinations to fabricate gratings on (llll 111 ) silicon. Their experimental results indicated that grating depths increased with the laser fluence for all treated samples. Additionally, the surface roughness increased with the laser fluence and pulse number for all polarization combinations. Oliveira et al. [11] used a Yb:KYW laser operated at a wavelength of $1030 \mathrm{~nm}$, 
a pulse width of $500 \mathrm{fs}$, and fluence levels between 0.5 and $2 \mathrm{~J} / \mathrm{cm}^{2}$ to irradiate titanium samples for generating micro/nano-periodic and nonperiodic ripple structures. Their experimental results revealed that a microcolumnar surface texture was developed in the center of the irradiated spots and that ripples were formed in the periphery of the spots. The dimensions of the ripple structures depended on the scanning speed; when the laser energy was less than the material ablation threshold, different micro-/nano-periodic structures could be produced. Angéline et al. [12] investigated the effect of laser surface treatment on the microstructure and mechanical properties of cast titanium alloys. A Nd:YAG laser welding machine with argon gas shielding was used to treat dumbbell- and plate-shaped cast titanium specimens. Microstructural and chemical analyses were performed using scanning electron microscopy (SEM), Xray diffraction (XRD), atomic-emission spectroscopy (AES), and Wavelength-dispersive spectroscopy (WDS). The XRD analysis indicated that beta phase formation was evident after the laser surface treatment process. Supplementary significant peaks of $\mathrm{TiO}, \mathrm{TiO}_{2}$, and $\mathrm{Ti}_{2} \mathrm{~N}$ were detected without argon gas shielding. When argon gas shielding was used, the presence of titanium oxide significantly decreased, and the peaks of titanium nitride disappeared. Coelho et al. [13] proposed three surface treatment methods for fabricating implant macrogeometries: electrodeposition, alumina-blasted/acid-etched treatment, and resorbable blasting media. Cylindrical, small healing, and large healing chamber configurations were used for implant surface characterization and biomechanical testing. Their experimental results revealed that the implant bulk design only affected results at 40 days in vivo. Bereznai et al. [14] used an ultrashort $\mathrm{KrF}$ excimer laser, operating in picoseconds, to treat medical implants. They tested biological behavior (osseointegration and biocompatibility) according to both the chemical composition and morphology of the surface. Modifications achieved using the excimer laser could improve surface characteristics to facilitate biointegration. Their experimental results showed that the chemical composition and morphology of the titanium surface were modified by the excimer laser treatment process. Effective polishing was achieved through homogeneous illumination with nanosecond laser pulses operated at a fluence of 3-5 $\mathrm{J} / \mathrm{cm}^{2}$. Because biological materials have different organizations, they must have a host bone and soft tissue for optimal surface compatibility and must have a mucous membrane exposure area with antimicrobial properties. Yoshinari et al. [15] summarized different surface modification methods for titanium using a cold-plasma technique. They reported that different surface modification methods produced inconsistent results in terms of surface bonding energy. The biological properties of titanium alloys subjected to surface treatment depend on film properties. Surface modification can improve the compatibility between biological tissue explants as revealed by the analysis of relevant characteristics. Elias et al. [16] proposed four groups, namely machined, acid, sandblasted, and anodized groups, for the surface treatment of unalloyed Ti (ASTM F67) in surgical implant applications. Serap et al. [17] used a fiber laser to treat the surfaces of specimens of commercially pure titanium dental implants. Four surface morphology patterns, namely honeycomb, spherical, diagonal, and moon patterns, were used. Their experimental results revealed that the honeycomb pattern was promising for balancing strain energy density with suspending interfacial loads, such as a spring system, which can serve as an efficient strain actuator. Milovanović et al. [18] used two lightemitting sources, namely $\mathrm{KrCl}(222 \mathrm{~nm})$ and $\mathrm{XeCl}(308$ $\mathrm{nm}$ ) excimer lasers, to modify the surface of titanium (Ti$6 \mathrm{Al}-4 \mathrm{~V}$ ) alloys. Their experimental results indicated that the $\mathrm{XeCl}$ excimer laser had a higher ablation rate and surface roughness than the $\mathrm{KrCl}$ excimer laser. Moreover, according to EDS analysis, they concluded that the XeCl excimer laser had the highest contribution (10\%) for oxide formation on the surface.

This study (Editor's and Reviewer \#2, Q2) investigated hybrid laser surface modification methods for a pure titanium foil by using a pulsed ultraviolet (UV) laser source (355 nm) combined with a high-speed galvanometric scanning unit. Due to the effect of surface energy, roughness, and residual height on contact angle; therefore, four surface treatment methods, namely nontreatment, microhole drilling, surface milling, and microhole drilling combined with surface milling, were proposed, and the effects of surface energy, roughness, and residual height on contact angle were examined. The characteristics of the surface-modified foil specimens were systemically analyzed using a threedimensional confocal laser scanning microscope to measure the surface micrograph and roughness of patterns. The contact angle variations between droplets on the textured surface were measured using an FTA 188 video contact angle analyzer.

\section{Experimental material and surface treatment laser system}

\subsection{Sample preparation of pure titanium foil (Editor's and Reviewer \#2, Q6)}

In this study, a pure titanium foil with a thickness of $100 \mu \mathrm{m}$ was used for the experiments. Before surface modification, the specimens were cleaned in an ultrasonic cleaner using a 75 vol. \% alcohol solution and 25 vol. \% distilled water. Figure. 1 shows the top-view SEM images of morphologies and energy-dispersive $\mathrm{X}$ ray spectroscopy (EDS) analysis results of the pure titanium foil. Table 1 summarizes the elements of the pure titanium foil. (a)

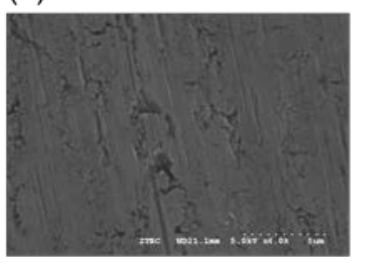

(c) (b)

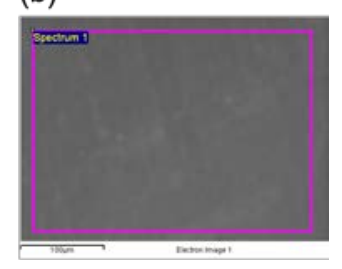

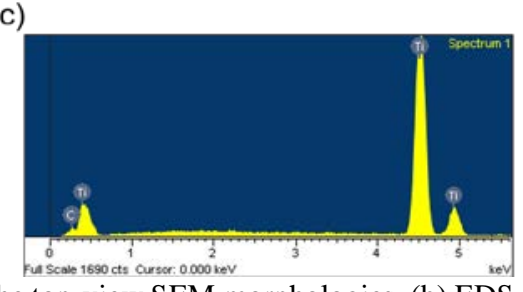

Fig. 1 (a) The top-view SEM morphologies, (b) EDS analysis 
region, and (c) EDS analysis of the pure titanium foil. (Editor's and Reviewer \#2, Q3)

Table 1 The elements of pure titanium foil.

\begin{tabular}{cc}
\hline Element & Atomic (\%) \\
\hline $\mathrm{C}$ & 15.03 \\
\hline $\mathrm{Ti}$ & 84.97 \\
\hline
\end{tabular}

\subsection{Surface modification using UV laser system}

A surface modification system comprising a UV laser source, galvanometric scanning unit, and servomotor driving stages was used in this study. This study also applied a diode-pumped solid-state UV laser source (Coherent, Inc. AVIA 355-14) with a wavelength of $355 \mathrm{~nm}$, maximum average output power of 14 watts, operating pulse repetition frequency range of $1-400 \mathrm{kHz}, \mathrm{TEM}_{00}$ transverse mode, and beam diameter of $3.5 \mathrm{~mm}$. Each pulsed width was of $32 \mathrm{~ns}$ at the $40 \mathrm{kHz}$ pulsed repetition rate.

A high-speed galvanometric system (Raylase AG model SS-15) with a focus shifter function could adjust the focus range in the $\mathrm{Z}$-axis direction from +15 to $-15 \mathrm{~mm}$ in order to conduct a helical drilling process. A telecentric scan lens with a focal length of $163 \mathrm{~mm}$ and scanning field of $60 \mathrm{~mm} \times 60 \mathrm{~mm}$ was used in this system.

\subsection{Three treatment methods for pure titanium foil}

All the (Editor's and Reviewer \#2, Q2) presented treatment method for pure titanium foil without any assist gas and machining environment under atmospheric conditions (i.e. the temperature of $25 \pm 1^{\circ} \mathrm{C}$ and humidity of $60 \pm 2 \%)$.

Case I- Nontextured specimens

Each titanium foil specimen had a square shape $(5 \times 5$ $\mathrm{mm}^{2}$ ) and thickness of $0.1 \mathrm{~mm}$, along with a grade of $99.67 \%$. The specimens were cleaned through ultrasonic treatment in a detergent solution, acetone, and 2-propanol, rinsed with deionized water, and finally dried in a compressed air flow.

Case II- Micro-hole-drilling method [19]

Commercially pure titanium foils, each with a thickness of $100 \mu \mathrm{m}$, were used in the experiments. Holes of various sizes were drilled into the pure titanium foil to investigate the effects of the drilling process on the characteristics (i.e., profile, oxidation, and residual properties) of the foil. The micro-hole-drilling method involves a three-step procedure outlined as follows [Figures 2 (a)-(c)]:

Step 1: The contour portion of the microholes was removed using a fluence laser beam to expedite the ablation rate of the material and reduce machining time [Figure 2(a)].

Step 2: The focus position of the focal lens was adjusted stepwise. High-fluence laser beams were used to trim residuals and ensure the roundness of the bottom circles of the microholes [Figure 2(b)].

Step 3: High-fluence laser beams were used to trim residuals and ensure the roundness of the top circles of the microholes [Figure 2(c)].

The relationship (Editor's and Reviewer \#2, Q2) between the micromachining parameters of microholes obtained using the UV laser drilling system on titanium alloys was studied to determine the cutting profile, oxidation, and residual properties of the microholes. These parameters could facilitate the fabrication of a $5 \times 5$ matrix of microholes with diameters of 200, 400, 600, and $800 \mu \mathrm{m}$.

Case III- Laser surface milling method

Figure 3 shows (Editor's and Reviewer \#2, Comments, Q2) the milling areas designed on the titanium foil. In the milling parameter evaluation, the milling area was set to $5 \mathrm{~mm}^{2}$. Each horizontal and vertical space had a dimension of $0.02 \mathrm{~mm}$. The pulse repetition frequency was set at $40 \mathrm{kHz}$, and the laser fluence levels were 19.7, 38.2, and $56.6 \mathrm{~J} / \mathrm{cm}^{2}$. Furthermore, the galvanometric scan speed ranged from 250 to $1500 \mathrm{~mm} / \mathrm{s}$. Table 2 summarizes the detailed laser milling parameters.

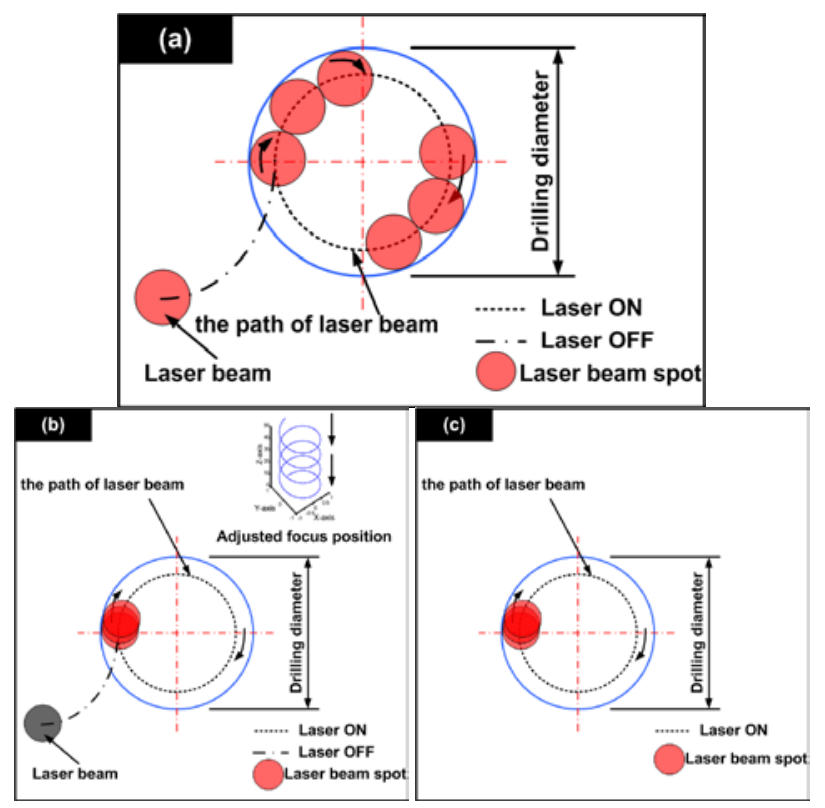

Fig. 2 Schematic diagram of the micro holes cut steps [19].
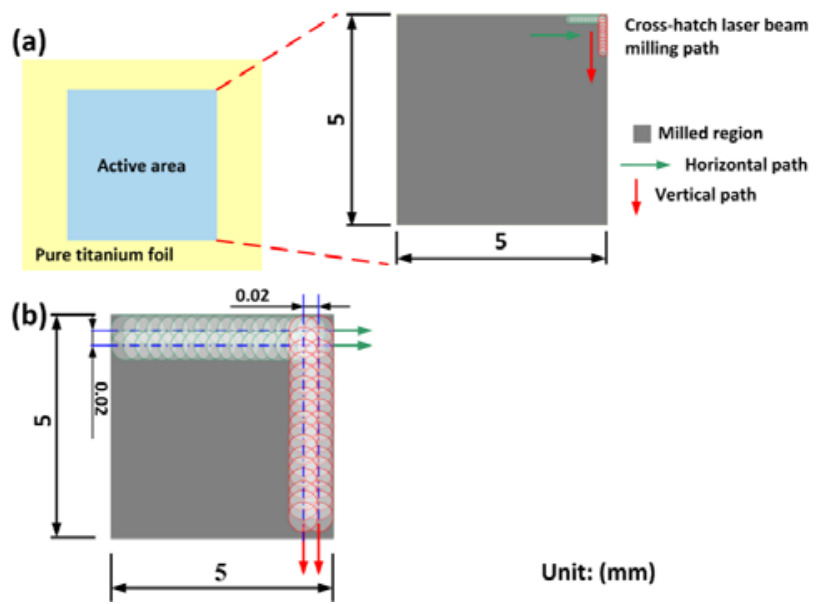

Fig. 3 Schematic diagram of the laser milling process.

Table 2 Parameters of laser milling of titanium foil.

\begin{tabular}{ccc}
\hline $\begin{array}{c}\text { Pulse repetition frequency } \\
(\mathrm{kHz})\end{array}$ & $\begin{array}{c}\text { Laser fluence } \\
\left(\mathrm{J} / \mathrm{cm}^{2}\right)\end{array}$ & $\begin{array}{c}\text { Scan speed } \\
(\mathrm{mm} / \mathrm{s})\end{array}$ \\
\hline & & 250 \\
40 & 19.7 & 500 \\
& 38.2 & 1000 \\
& 56.6 & 1500 \\
\hline
\end{tabular}

Case IV- Hybrid method combining micro-hole-drilling and laser milling methods 
The hybrid laser surface modification method, involving a combination of the micro-hole-drilling and laser milling methods, was also used in the experiments. The microhole diameters were 200, 400, 600, and $800 \mu \mathrm{m}$. For laser milling parameters, each horizontal and vertical space had a dimension of $0.02 \mathrm{~mm}$. The pulse repetition frequency was set at $40 \mathrm{kHz}$. The galvanometric scan speed was fixed at $1000 \mathrm{~mm} / \mathrm{s}$.

\subsection{Characterization analysis of modified titanium foils} (Editor's and Reviewer \#2, Q4)

The surface morphology and residual height of the modified titanium alloy foils were recorded using a threedimensional confocal laser scanning microscope (KEYENCE, VK-9700), and analytical software (KEYENCE, VK-Analyzer Plus ${ }^{\mathrm{TM}}$ ) based on the ISO-4287 standard was used to evaluate the machined surface roughness. The contact angle differences between droplets on the textured surface were measured using the FTA 188 video contact angle analyzer. The contact angle $\left(\theta_{c}\right)$ is defined as the angle formed by the intersection of liquid-solid and liquid-vapor interfaces (Figure 4). The magnitude of solid surface wetting induced by a liquid can be evaluated using the contact angle $\left(\theta_{c}\right)$, which can be defined using Young's equation (1):

$$
\gamma_{S G}=\gamma_{S L}+\gamma_{L G} \cos \theta_{c}
$$

where $\gamma_{\mathrm{SG}}, \gamma_{\mathrm{SL}}$, and $\gamma_{\mathrm{LG}}$ represent (Editor's and Reviewer \#2, Q2) the surface tensions of the solid-gas, solid-liquid, and liquid-gas interfaces, respectively.

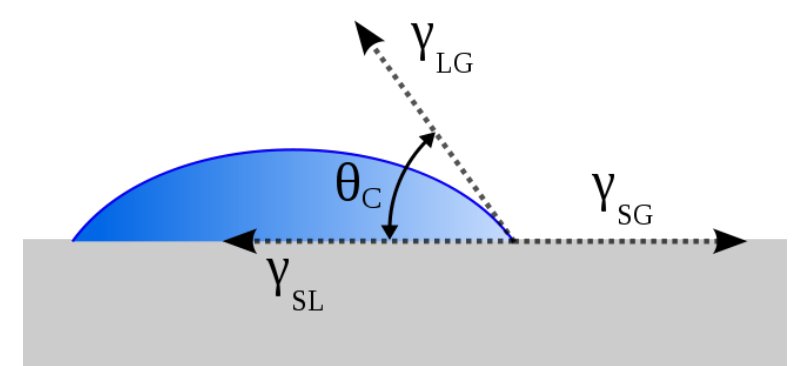

Fig. 4 Schematic of contact angle formed by sessile liquid drops on a smooth homogeneous solid surface.

The relationship (Editor's and Reviewer \#2, Q2) between roughness and wettability was defined by Wenzel, who stated that adding surface roughness enhances the wettability caused by the chemistry of the surface. For example, if the surface is chemically hydrophobic, it becomes even more hydrophobic with increased surface roughness. Wenzel's statement can be described by equation (2) [20]:

$$
\cos \theta_{W^{*}}=r \cos \theta
$$

where $\theta_{\mathrm{W}^{*}}, \theta$, and $\mathrm{r}$ denote the measured contact angle, Young's contact angle, and roughness ratio, respectively. The roughness ratio is defined as the ratio between the actual solid surface area and projected solid surface area. This law predicts that the experimental contact angles increase with roughness if $\theta>90^{\circ}$ and predicts the opposite trend if $\theta<90^{\circ}$

According to (Editor's and Reviewer \#2, Q2) the Figure 4 and Eq. (1), the system energy is tightly associated with surface tension and adhesion. When the larger $\gamma_{\mathrm{SG}}$, the more hydrophilic the surface, the larger surface energy [21].

\section{Results and discussion}

\subsection{Surface morphology and residual height measure- ment}

Figure 5 depicts the top-view SEM images of laser microholes, with a diameter of $200 \mu \mathrm{m}$, drilled on the titanium foil. The figure reveals slag phenomena, which can be explained as follows: Thermal diffusion and thermal conductivity induced by a laser during surface drilling result in the melting of substances within the material, and such substances can prompt the effluence of debris from the hole, which can contaminate surrounding drilling areas. In addition, long irradiation time generates cumulative energy within the material. Splashing debris around the drilled holes results in the formation of a recast layer called slag. Surface debris can form slag residues on the surface, affecting the final morphological properties. The top-view SEM images of the pure titanium foil specimens indicate that when the specimens were subjected to laser surface treatment [Figure 6(a)], the specimen materials melted and resolidified on the surface [Figure 6(b)]. Figure 7 illustrates the residual heights of the areas surrounding the drilled holes for different hole diameters. As revealed in the plot, the residual height decreased as the hole diameter increased.

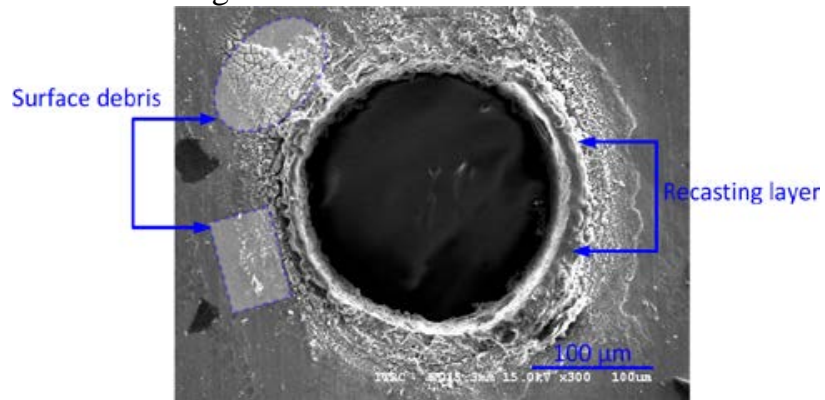

Fig. 5 The SEM top view image of laser micro-hole drilling on titanium foil.
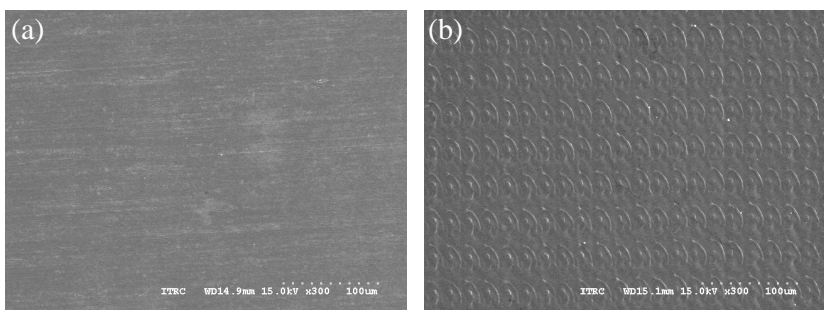

Fig. 6 The SEM top view image of the (a) untreated and (b) laser milling process on the pure titanium foil.

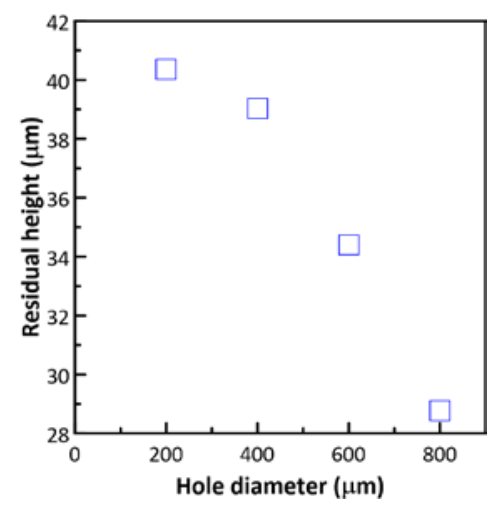

Fig. 7 Relationship between micro hole diameter and residual height on titanium foil. 


\subsection{Surface roughness evaluation}

Figures 8(a), 8(b), 8(c), and 8(d) (Editor's and Reviewer \#2, Q2) depict the surface morphologies of specimens subjected to no treatment (pure titanium foil), laser hole drilling, laser milling, and hybrid laser surface treatment, respectively. The roughness of the treated region was evaluated on the basis of the ISO-4287 standard. Regarding laser milling, Figure 9(a) presents a plot of the relationship between different scanning speeds and surface roughness levels for the titanium foil. Laser milling was performed on the titanium foil by using laser fluence levels of 19.7, 38.2, and $56.6 \mathrm{~J} / \mathrm{cm}^{2}$ and a pulse repetition rate of $40 \mathrm{kHz}$. As indicated in the plot, the surface roughness decreased as the scanning speed increased. Regarding laser microhole drilling, the surface roughness increased with the microhole diameter [Figure 9(b)]. (a)

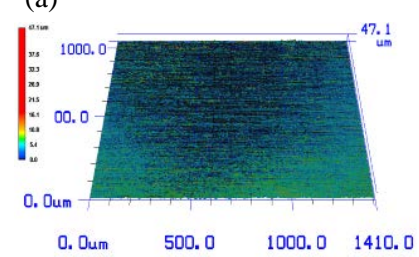

(c)

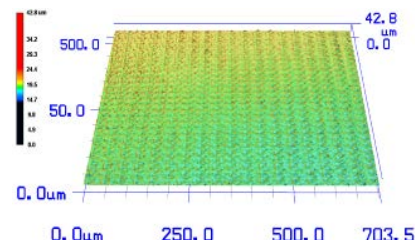

(b)

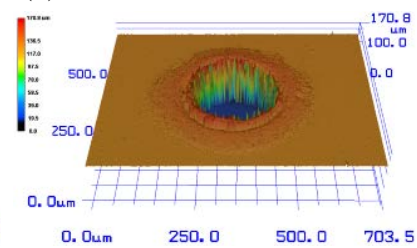

(d)

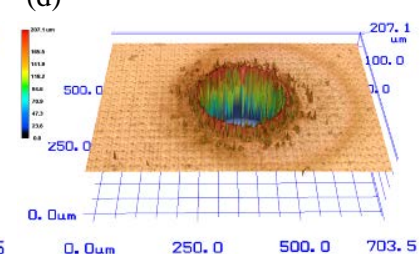

Fig. 8 3D surface morphology image (a) pure titanium foil, (b) laser hole drilling, (c) laser milling, (d) hybrid laser surface treatment.

(a)

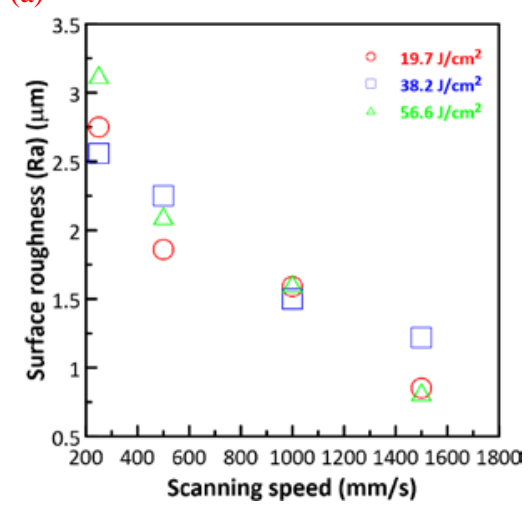

(b)

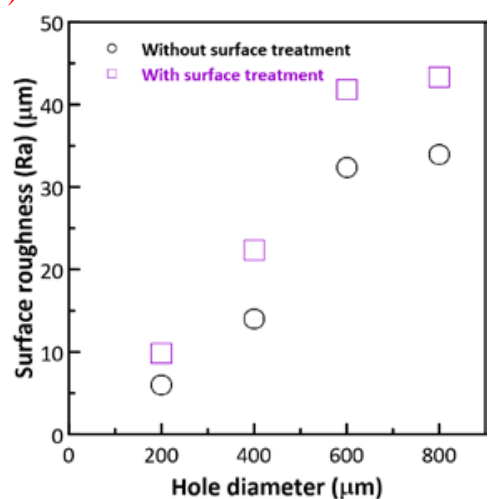

Fig. 9 Relationship of (a) different scanning speed versus surface roughness and (b) different drilled hole diameter versus surface roughness of the treated titanium foil. (Editor's and Reviewer \#2, Q5)

\subsection{Contact angle measurement evaluation}

\subsubsection{Effects of hole diameter on contact angle}

Droplet experiments were conducted to describe the wettability characteristics of the textured surfaces used in this study. In these experiments, a $3-\mu \mathrm{L}$ droplet was gently placed on the textured surface by using a micropipette. Droplet shapes were captured to measure the apparent contact angle by using the FTA 188 video contact angle analyzer. Figure 10 illustrates the relationship between contact angle and microhole diameter for the different laser modification methods performed on the titanium foil surface. The experimental data revealed that the contact angle decreased as the microhole diameter increased for the first three methods except for the laser milling method. By contrast, the contact angle on the titanium foil surface increased with the microhole diameter for the hybrid method combining laser drilling and milling.

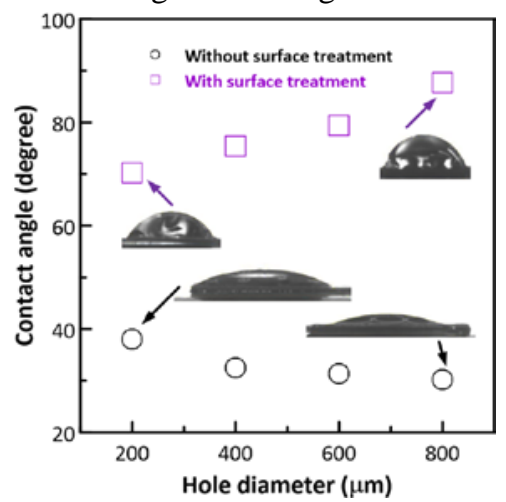

Fig. 10 Relationship of different drilled hole on the contact angle of the titanium foil.

\subsubsection{Effects of hole diameter on contact angle}

Figure 11 shows the effects of scanning speed on contact angle for the laser milling method. As shown by the experimental data, the effects of contact angle at different scanning speeds were not significant when the laser energy levels were 19.7 and $38.2 \mathrm{~J} / \mathrm{cm}^{2}$. However, the effect of the contact angle at different scanning speeds was significant when the laser energy increased to $56.6 \mathrm{~J} / \mathrm{cm}^{2}$. Furthermore, a satisfactory hydrophilic (Reviewer \#1, Q1) quality (contact angle of $12.57^{\circ}$ ) of the foil surface was obtained with milling parameters of $56.6 \mathrm{~J} / \mathrm{cm}^{2}$ for laser energy and 1500 $\mathrm{mm} / \mathrm{s}$ for scanning speed.

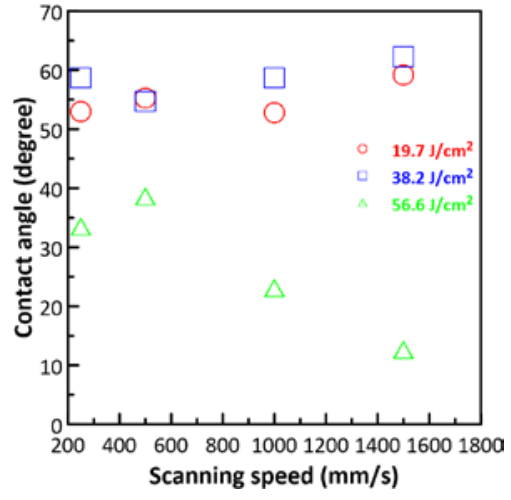


Fig. 11 Relationship of different scanning speed on the contact angle of the titanium foil.

\subsubsection{Effects of surface roughness on contact angle}

This study examined the effects of surface roughness on contact angle at different milling speeds. Therefore, when the laser fluence was set at $56.6 \mathrm{~J} / \mathrm{cm}^{2}$ and the milling speeds were varied, distinct relationships between surface roughness and contact angle could be obtained. Figure 12 illustrates the relationship between surface roughness and contact angle at different laser milling speeds for pure titanium foil specimens. The experimental results indicated that the surface roughness ranged from 0.82 to $3.13 \mu \mathrm{m}$. A surface with a roughness of $0.82 \mu \mathrm{m}$ and contact angle of $12.57^{\circ}$ was considered to exhibit superior hydrophilic properties to other surfaces. Based on the Evgeny et al. [22] experimental results, the rougher surfaces have higher surface energy.

Figure 13 shows the relationship between surface roughness and contact angle for the different surface modification methods. As revealed by the experimental results, the hybrid surface treatment method was associated with a larger contact angle than the micro-hole-drilling method.

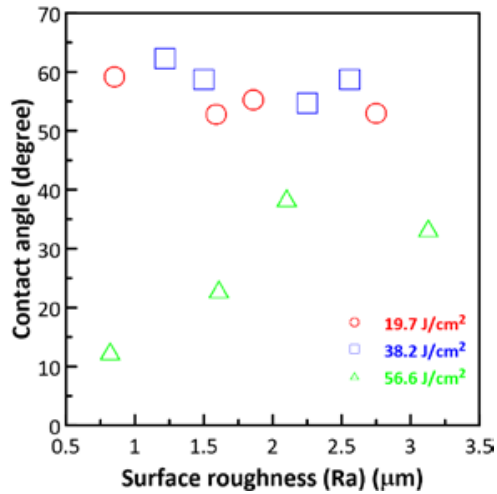

Fig. 12 Relationship of different surface roughness of laser fluence on the contact angle of the titanium foil.

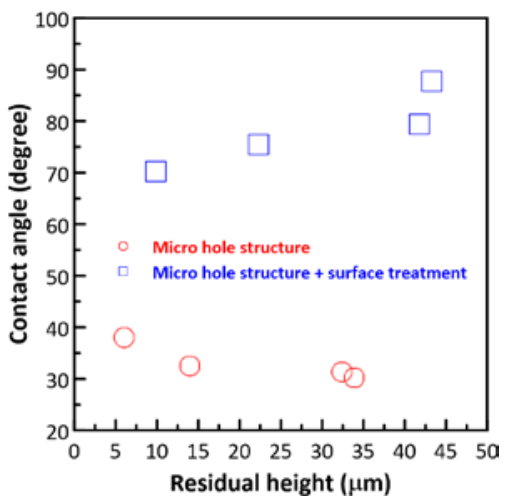

Fig. 13 Relationship of different surface roughness of drilled hole on the contact angle of the titanium foil.

\subsubsection{Effects of residual height on contact angle}

Figure 14 shows the relationship between residual height and contact angle. The experimental results indicated that the contact angle increased with the residual height. When the hole diameter and residual height were $800 \mu \mathrm{m}$ and $28.76 \mu \mathrm{m}$, respectively, the contact angle was $30.18^{\circ}$. This result is better than laser milling surface hydrophilic properties.

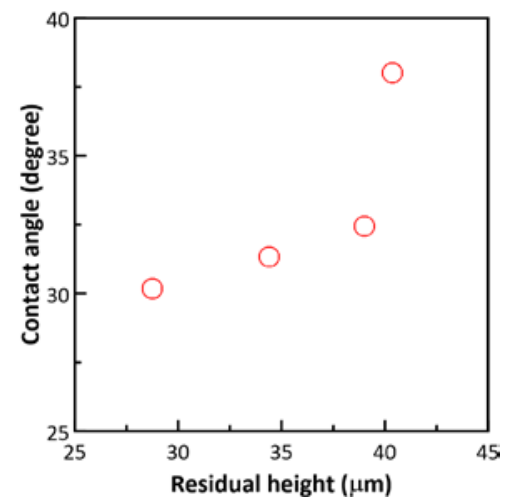

Fig. 14 Relationship of different residual height on the contact angle of the titanium foil.

\section{Conclusions}

This study applied (Editor's and Reviewer \#2, Q2) a high-frequency Nd:YVO 4 UV laser combined with a highspeed galvanometric scanning unit and operated using different parameters, including laser energy and scanning speed, for the surface modification of titanium foil specimens. The results of this study are summarized as follows:

(a) Regarding laser microhole drilling, the residual height around microholes and the contact angle of the foil surface decreased as the microhole diameter increased. The contact angle of the foil surface decreased from $56^{\circ}$ to $30.18^{\circ}$, and the surface property changed from hydrophobic to hydrophilic when the microhole diameter was $800 \mu \mathrm{m}$.

(b) Regarding laser milling, in this study, the optimal laser fluence and scanning speed of $56.6 \mathrm{~J} / \mathrm{cm}^{2}$ and $1500 \mathrm{~mm} / \mathrm{s}$, respectively, provided a contact angle of $12.57^{\circ}$, signifying satisfactory hydrophilic quality.

(c) The measured contact angle was between $70^{\circ}$ and $88^{\circ}$, revealing a hydrophobic quality, when the hybrid laser drilling and milling method was used. This study successfully used a high-frequency $\mathrm{Nd}: \mathrm{YVO}_{4} \mathrm{UV}$ laser combined with a high-speed galvanometric scanning unit for the surface modification of titanium foil specimens. This method can be applied in research related to the adhesive quality of biological cells in the future.

\section{Acknowledgement}

The authors thank (Editor's and Reviewer \#2, Q2) the Ministry of Science and Technology of Taiwan for financially supporting this research under contract nos. MOST 104-2221-E-492-039 and MOST 104-2622-E-492-008CC3. Measuring supports from Ms. Nien-Nan Chu of Instrument Technology Research Center. Moreover, this manuscript was edited by Wallace Academic Editing.

\section{References (Chief Editor's comment, Q2; Editor's} and Reviewer \#2, Q1)

[1] D.G. Olmedo, G. Duffó, R.L. Cabrini, M.B. Guglielmotti: Int. J. Oral Maxillofac. Surg., 37, (2008) 1032.

[2] R.M. Wazen, L.P. Lefebvre, E. Baril, A. Nanci: J. Biomed. Mater. Res. B: Appl. Biomater., 94, (2010) 64.

[3] Z. Yoshimitsu, A. Nakajima, T. Watanabe, K. Hashimoto: Langmuir., 18, (2002) 5818.

[4] J. Lee, B. He, N.A. Patankar: J. Micromech. Microeng., 15, (2005) 591. 
[5] H. Schulz, M. Leonhardt, H.J. Scheibe, B. Schultrich: Surf. Coat. Technol., 200, (2005) 1123.

[6] M. Miwa, A. Nakajima, A. Fujishima, K. Hashimoto, T. Watanabe: Langmuir., 16, (2000) 5754.

[7] R. Kannan, D. Sivakumar: Colloids Surf. A., 317, (2008) 694.

[8] M. Groenendijk: Laser Technol. J., 3, (2008) 44.

[9] H.Y. Zheng, W. Zhou, H.X. Qian, T.T. Tan, G.C. Lim: Appl. Surf. Sci., 236, (2004) 114.

[10] C.W. Hee, B.K.A. Ngoi, L.E.N. Lim, K. Venkatakrishnan, W.L. Liang: Opt. Laser Tech., 37, (2005) 93.

[11] V. Oliveira, S. Ausset, R. Vilar: Appl. Surf. Sci., 255, (2009) 7556.

[12] A.P. Quintin, I. Watanabe, E. Watanabe, C. Bertrand: Dent. Mater., 28, (2011) 945.

[13] P.G. Coelho, R. Granato, C. Marin, H.S. Teixeira, M. Suzuki, G.B. Valverde, M.N. Janal, T. Li, E.A. Bonfante: J. Mech. Behav. Biomed. Mater., 284, (2011) 1974.

[14] M. Bereznai, I. Pelsöczi, Z. Tóth, K. Turzó, M. Radnai, Z. Bor, A. Fazekas: Biomaterials, 24, (2003) 4197.

[15] M. Yoshinari, K. Matsuzaka, T. Inoue: Jpn. Dent. Sci. Rev., 47, (2011) 89.

[16] C.N. Elias, Y. Oshida, J.H.C. Lima, C.A. Muller: J. Mech. Behav. Biomed. Mater., 23, (2008) 234.

[17] S. Çelen, H. Özden: Appl. Surf. Sci., 263, (2012) 579.

[18] D.S. Milovanović, S.M. Petrović, M.A. Shulepov, V.F. Tarasenko, B.B. Radak, Š.S. Miljanić, M.S. Trtica: Opt. Laser Tech., 54, (2013) 419.

[19] W.T. Hsiao, S.F. Tseng, K.C. Huang, D. Chiang, M.F. Chen: Applied Physics A., 110, (2013) 565.

[20] R.N. Wenzel: Ind. Eng. Chem., 28, (1936) 988.

[21] K.C. Yung, W.J. Wang, T.Y. Xiao, H.S. Choy, X.Y. Mo, S.S. Zhang, Z.X. Cai: Surf. Coat. Technol., 351, (2018) 89.

[22] B. Evgeny, T. Hughes, D. Eskin: Corros. Sci., 103, (2016) 196.

(Received: June 29, 2018, Accepted: October 13, 2018) 